\title{
De las nanobiomoléculas a la nanobiología y nanomedicina
}

\author{
Natalia Oddone, Ana Zambrana, Verónica Bervejillo, \\ Andrés Alberro, Inés Rauschert, María Bausero, \\ Mariel Flores, Marcos Tassano, Pablo Cabral, Juan Claudio Benech*
}

ResUmen: Actualmente, estamos en una etapa en la que las ciencias biomédicas buscan mejorar el diagnóstico y la terapia de varias enfermedades, por medio del empleo de novedosos dispositivos a escala nanométrica. Micelas poliméricas, liposomas, dendrímeros y nanopartículas biodegradables, son algunos ejemplos de nanomateriales que se investigan en los laboratorios, que están en fase pre-clínica o ya se emplean en la clínica. En este momento se cuenta con numerosos nuevos sistemas nanoestructurados con múltiples y potenciales aplicaciones en el área biomédica. Sin embargo, a pesar de que en muchos casos se han realizado caracterizaciones fisicoquímicas de los mismos, no se ha estudiado su interacción con diferentes sistemas biológicos y con las nanobiomoléculas que los componen.

En este artículo, mostramos la evolución del trabajo que estamos llevando a cabo en nuestro laboratorio. En primera instancia, describimos las investigaciones que hemos realizado estudiando diferentes nanobiomoléculas. Nuestro objetivo es intentar entender la señalización y regulación de procesos biológicos como la síntesis de proteínas en el sistema nervioso o la síntesis de ARN en el núcleo celular. En segunda instancia, describimos estudios que estamos realizando utilizando dendrímeros y analizando sus posibles aplicaciones en el tratamiento de enfermedades como el parto prematuro, el cáncer o la diabetes. Por último, describimos brevemente la microscopía de fuerza atómica y sus aplicaciones en ciencias biológicas y biomédicas, finalizando con un ejemplo concreto de nuestra investigación.

PaLABRAS CLAVES: nanobiomoléculas, nanomedicina, dendrímeros, microscopía de fuerza atómica

ABSTRACT: We are nowadays in a new stage in which biomedicine searches the ways to improve diagnosis and therapy of several diseases by using novel nanodevices. Polymeric micelles, liposomes, dendrimers, biodegradable nanoparticles and others are examples of nanoparticulate materials researched in laboratories, undergoing preclinical development, or already used in the clinic. At present, we have numerous nanoestructured systems with multiple potential applications in the biomedical area. Nevertheless, in spite of the fact that in many cases physicochemical characterizations of the mentioned nanosystems were carried out, their interactions with the biological systems or the nanobiomolecules that compose these systems were not studied.

In this article, we show the research performed in our laboratory. First, we describe studies we have carried out studying different nanobiomolecules. By these experiments we are trying to understand signaling and regulation of biological processes such as protein synthesis in the nervous system or RNA synthesis in the cellular nucleus. Second, we describe experiments we are performing using dendrimers and studying their possible applications to treat diseases such as premature labor, cancer or diabetes. Finally, we briefly describe atomic force microscopy applications in biological and biomedical sciences and give a concrete example of our work.

KEY WORDS: nanobiomolecules, nanomedicine, dendrimers, atomic force microscopy.

\footnotetext{
* Director del Laboratorio de Señalización Celular y Nanobiología, Instituto de Investigaciones Biológicas Clemente Estable (IIBCE). Av. Italia 3318, CP 11600, Montevideo, Uruguay, Tel. +5982-24871616, int. 107. Correo electrónico: benech@iibce.edu.uy, o juanclaudio.benech@gmail.com
} 


\section{INTRODUCCIÓN}

Para poder comprender bien los conceptos de nanociencia, nanotecnología, nanobiología y nanomedicina y poder dar una definición de los mismos, es conveniente establecer primero cuáles son las principales diferencias entre ciencia y tecnología. De una manera general, podemos decir que la ciencia es el trabajo que se realiza en un laboratorio de investigación, aplicando el método científico, en búsqueda de respuestas para determinadas preguntas. Los resultados obtenidos de estas investigaciones, se traducen en trabajos científicos que se publican en revistas especializadas internacionales. Estos trabajos, son previamente analizados por otros científicos especialistas en el tema en cuestión. La tecnología parte de los conocimientos básicos establecidos por la ciencia, para construir un dispositivo o un aparato que tenga una utilidad determinada. El conocimiento necesario para generar el nuevo dispositivo o aparato, generalmente da origen a una patente (mecanismo utilizado para proteger la invención y los derechos de los inventores). La creación de tecnología, proporciona a los científicos nuevas herramientas para contestar nuevas preguntas y avanzar en la ciencia, que a su vez generará nueva tecnología. Por lo tanto, podríamos decir que ciencia y tecnología son las dos caras de una misma moneda.

El prefijo "nano" significa la mil millonésima parte de algo. En el caso de un nanómetro, significa la mil millonésima parte de un metro, es decir $10^{-9} \mathrm{~m}$. Dado que muchas de las propiedades físicas y químicas de la materia se modifican en tamaños comprendidos entre 0.1 y 100 nanómetros, éste sería el mundo "nano" estrictamente hablando.

En el año 1959, varios años antes de recibir el Premio Nóbel de Física, el Dr. Richard Feynman pronunció en el Instituto de Tecnología de California una conferencia titulada "Hay mucho espacio al fondo" (There is plenty of room at the bottom). En esta conferencia, el Dr. Feynman puso los pilares de lo que luego sería conocido como nanotecnología. En su charla, Feynman expuso sus ideas de cómo manipular, controlar y fabricar objetos de muy pequeñas dimensiones. Decía Feynman en su discurso: "Los principios de la física, tal como yo los veo, no niegan la posibilidad de manipular las cosas átomo por átomo. Al no violar ninguna ley, no hay motivo para que no pueda hacerse". "En el mundo de lo muy, muy pequeño, muchas cosas nuevas podrán suceder, porque los átomos se comportan de manera distinta a como lo hacen los objetos a mayor escala, pues deben satisfacer las leyes de la mecánica cuántica". Y continúa... "A nivel atómico, aparecen nuevos tipos de fuerza, nuevas posibilidades, nuevos efectos". Ésta es una de las ideas básicas de la nanotecnología, que consiste en un cambio de estrategia a la hora de fabricar estructuras: el paso de una tradición de arriba hacia abajo top-down a un futuro de abajo hacia arriba bottom-up, manipulando la materia a nivel atómico.

Podemos dar ahora una definición entendible del concepto nanotecnología: es el estudio, síntesis y manipulación de materiales y sistemas funcionales a través del control de la materia a escala nanométrica; tiene como objetivo la fabricación de materiales, objetos y dispositivos tecnológicos a esa escala. Por otra parte, la nanociencia estudia la materia a escala de estructuras moleculares y atómicas, utilizando las herramientas de la física, química, biología y ciencia de los materiales. De esta forma busca responder preguntas básicas sobre el comportamiento de estructuras a esa escala.

Muchos componentes biológicos como el ADN, las membranas celulares, las proteínas, y las estructuras biológicas como los ribosomas o los complejos de poros 
nucleares tienen tamaño nanométrico. Como todas las células eucariotas están formadas por estos componentes comunes, podemos decir que en la biología hay "vida en el nanomundo".

Una razón para estudiar la biología a nanoescala, es poder observar propiedades que no se ven en escalas micro o macro. Por ejemplo, medir propiedades físicas de proteínas individuales nos puede dar información sobre su estructura y función. Esta información sin duda nos ayudará a entender cómo los diferentes componentes de un sistema biológico trabajan juntos.

La nanobiología, podría ser definida como el estudio del material biológico a escala nanométrica. Sin embargo, los biólogos moleculares han estado trabajando con nanobiomoléculas desde hace varias décadas. Por lo tanto, estrictamente hablando, se considera nanobiología la disciplina que utiliza productos y conocimiento que surgen de la nanotecnología y los aplica a la investigación en biología. Sería la fusión de la investigación biológica con la nanotecnología, así como la nanomedicina se puede definir como el uso de nanotecnología en el campo médico. (Por consulta de publicaciones recientes ver Balogh, 2010; Kroll, 2012, Jain, 2012.).

A continuación daremos algunos ejemplos sobre el trabajo de investigación que estamos desarrollando en nuestro laboratorio, en el Instituto de Investigaciones Biológicas Clemente Estable. El primer ejemplo, corresponde a las investigaciones que hemos realizado con nanobiomoléculas. El eje de estas investigaciones es el rol del ion $\mathrm{Ca}^{2+}$, su transporte y la regulación de eventos trascendentes como la síntesis proteica en terminales neuronales presinápticos o la regulación de la síntesis de ARN. El segundo ejemplo, corresponde a las investigaciones en curso donde utilizamos nanopolímeros artificiales llamados dendrímeros, donde estudiamos sus posibles aplicaciones como transportadores de drogas o agentes de imagenología en diferentes sistemas biológicos. Por último, nos vamos a referir al microscopio de fuerza atómica y las investigaciones que estamos realizando en el área de las ciencias biológicas y biomédicas. Dado que en los últimos dos ejemplos estamos incluyendo en nuestras investigaciones productos surgidos de la nanotecnología, podemos decir que son investigaciones genuinas en nanobiología y nanomedicina.

\section{TRABAJOS DE INVESTIGACIÓN}

\section{Señalización celular y nanobiomoléculas}

Nuestro laboratorio se dedica al estudio de las señales celulares. La transducción de señales es el conjunto de procesos o etapas que ocurren de forma concatenada, por el cual una célula convierte una determinada señal o estímulo exterior, en otra señal o respuesta específica. El proceso de transmisión de señal afecta a una secuencia de reacciones bioquímicas dentro de la célula. Esto se lleva a cabo a través de proteínas o enzimas unidas a otras sustancias llamadas segundos mensajeros. Los cambios en la concentración citosólica de $\mathrm{Ca}^{2+}$ han mostrado ser un sistema de señalización muy versátil. Este sistema regula varios procesos celulares diferentes como la motilidad, secreción y la proliferación celular, entre otros. Los cambios en la concentración intracelular de $\mathrm{Ca}^{2+}$ son detectados por las células como una señal con un significado que debe ser decodificado. Esta decodificación de la información contenida en las señales de $\mathrm{Ca}^{2+}$ es mediada por proteínas capaces de unir $\mathrm{Ca}^{2+}$ (sensoras de $\mathrm{Ca}^{2+}$ ) que transmiten esta información al blanco final. Como forma de garantizar la función de señaliza- 
ción del $\mathrm{Ca}^{2+}$, las células cuentan con varios sistemas de transporte de dicho ion, que regulan su homeostasis en forma muy precisa (Sotelo y Benech, 1997). Uno de estos sistemas de transporte, son las llamadas bombas de $\mathrm{Ca}^{2+}\left(\mathrm{Ca}^{2+} \mathrm{Mg}^{2+}-\mathrm{ATPasas}\right)$. Estas bombas son proteínas y por lo tanto nanoestructuras biológicas.

Trabajando con este transportador hemos obtenido aportes relevantes. Encontramos evidencias de que en ciertas condiciones experimentales, la bomba de $\mathrm{Ca}^{2+}$ puede comportarse como un canal de $\mathrm{Ca}^{2+}$ (Benech et al., 1991, 1995; García-Teijeiro et al., 1999). Caracterizamos el ciclo de reacciones de la $\mathrm{Ca}^{2+}$ ATPasa del sistema tubular denso de plaquetas humanas y mostramos que la estequiometría de esta SERCA (Sarco Endoplasmic Reticulum Calcium ATPase) es diferente a la SERCA de retículo sarcoplásmico de músculo esquelético de conejo (Benech et al., 1995). También mostramos la capacidad de ciertas drogas de desacoplar la enzima y promover un aumento en el eflujo de $\mathrm{Ca}^{2+}$ pero sin síntesis de ATP (García-Teijeiro et al., 1999).

Por otra parte, en colaboración con el Dr. J. R. Sotelo, hemos trabajado en aspectos metabólicos del sistema nervioso, especialmente en la síntesis proteica en el territorio axonal, sináptico y su regulación. En los, organismos eucariotas la síntesis de proteínas extra mitocondriales ocurre en nanoestructuras biológicas denominadas ribosomas. Éste es un tema de investigación muy interesante e importante, ya que en los últimos años han aumentado las evidencias de la existencia de ribosomas y polisomas activos en el dominio axonal y presináptico. De acuerdo con el dogma establecido en las células nerviosas todas las proteínas son sintetizadas en el soma de la neurona y luego transportadas por transporte axonal, al axón y al terminal nervioso (véase Ochs, 1982). Sin embargo, varios grupos de investigación (Dr. E. Koenig, Universidad de Buffalo, USA; Dr. A. Giuditta, Universidad de Nápoles, Italia; Dr. Jaime Álvarez, Universidad Católica de Chile), incluyendo el nuestro, han presentado evidencias muy convincentes que apoyan la existencia de un Sistema Local de Síntesis de Proteínas en axones y terminales nerviosas. En este sentido, hemos contribuido con varias publicaciones utilizando diversas técnicas como auto radiografía a nivel de microscopía óptica, inmunocitoquímica a nivel óptico y electrónico, inmunoprecipitación, Northern blot, RT-PCR, inmunoblot, etc. (Sotelo y Benech, 1997; Sotelo et al., 1999; Sotelo-Silveira et al., 2000; Calliari et al., 2002). (Véase también Koenig y Giuditta, 1999 y Álvarez et al., 2000).

Con respecto al terminal nervioso, al inicio de la década de los 90, se reportó que la fracción sinaptosomal obtenida del lóbulo óptico del calamar fue capaz de sintetizar proteínas extra-mitocondriales (Crispino et al., 1993). Esta fracción fue caracterizada por técnicas bioquímicas y de auto radiografía a nivel de microscopía electrónica. Se encontró que la fracción corresponde al componente presináptico de las neuronas fotorreceptoras del lóbulo óptico del calamar, el cual contiene polisomas activos (Crispino et al., 1997). La caracterización de la existencia de síntesis local de proteínas a nivel axonal y del terminal nervioso, nos llevó a estudiar posibles mecanismos de regulación y uno de los candidatos obvios era el $\mathrm{Ca}^{2+}$. En este sentido, en colaboración con el Dr. A. Giuditta comenzamos a estudiar la modulación del sistema local de síntesis proteica en los sinaptosomas preparados del lóbulo óptico del calamar. Observamos que este proceso estaba muy próximo de su máximo, a la concentración basal de $\mathrm{Ca}^{2+}$ citosólico y era marcadamente inhibida cuando la concentración de este ión era disminuida o aumentada (Benech et al., 1994, 1996, 1997, 1999). La posibilidad de que el $\mathrm{Ca}^{2+}$ module la síntesis local de proteínas sinaptosomal, fue confirmada midiendo la concentración de $\mathrm{Ca}^{2+}$ en un único sinaptosoma (figura 1), previamente cargado 
con Fura-2-acetoximetil éster (FURA2 AM). Estos experimentos fueron realizados en colaboración con el Dr. E. Brown del Laboratorio de Neurobiología de la Estación Zoológica de Nápoles, Italia. En su estado de reposo, la concentración de $\mathrm{Ca}^{2+}$ fue de $80 \mathrm{nM}$ $(\mathrm{n}=10)$. La adición de cafeína, tapsigargina y $\mathrm{KCl}$, aumentaron la concentración de $\mathrm{Ca}^{2+}$ a $300 \mathrm{nM}$. Por otra parte, el quelante de $\mathrm{Ca}^{2+}$ BAPTA AM disminuyó la concentración citosólica de dicho ion a menos de $100 \mathrm{nM}$ (Benech et al., 2000). El conjunto de estos resultados sugiere que el rango de sensibilidad de modulación de la síntesis proteica local está en una "ventana" de $\mathrm{Ca}^{2+}$ entre 80 y 300 nM. Estos resultados sugieren que la nanomaquinaria de síntesis proteica eucariota (ribosomas) está presente a nivel presináptico. Los resultados también sugieren, que variaciones en la concentración del $\mathrm{Ca}^{2+}$ citosólico en el terminal presináptico, producto de la actividad neuronal, pueden contribuir a la modulación de la síntesis local de proteínas con importancia para procesos plásticos neuronales como la memoria o el aprendizaje. Por último, los resultados también sugieren la existencia de poblaciones de ARNm específicas a nivel del terminal nervioso, aspecto que llevó a interesarnos en el proceso de síntesis de ARN a nivel del núcleo celular y su regulación.

FIGURA 1. A: Microscopía láser confocal de un sinaptosoma típico obtenido del lóbulo óptico del calamar en agua artificial de mar (imagen de transmisión). B: Imagen confocal de fluorescencia del mismo sinaptosoma incubado previamente con Fluo-3. La señal de fluorescencia fue fijada al máximo con propósitos ilustrativos. Barra de escala, $2 \mu \mathrm{m}$.
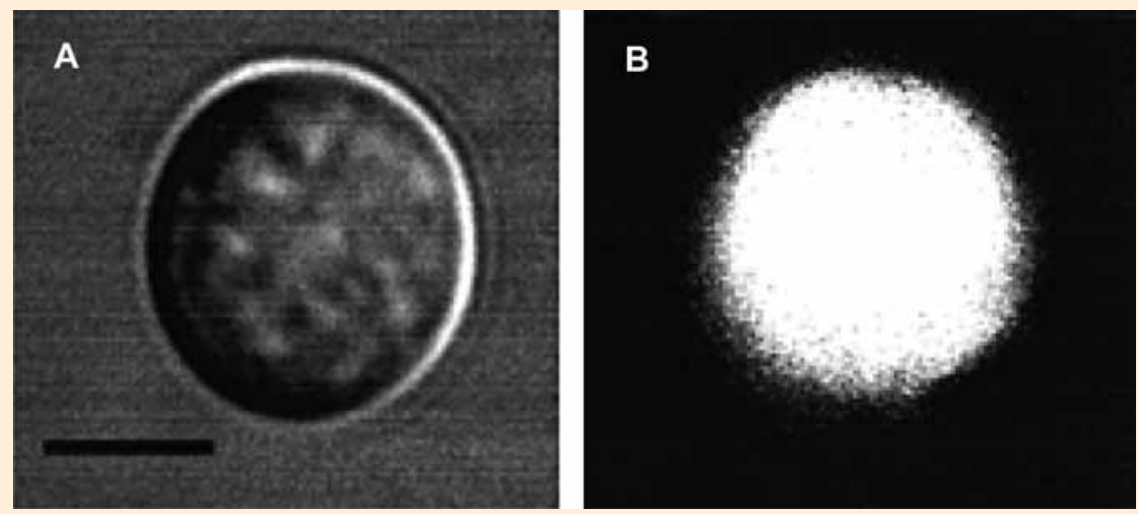

En los últimos años, el papel del $\mathrm{Ca}^{2+}$ como segundo mensajero se ha expandido a una gran variedad de eventos, desde mecanismos celulares de transducción ya clásicos, como lo es el acoplamiento excitación-contracción en las células musculares, a fenómenos cognitivos tan complejos como el aprendizaje y la memoria. En este contexto, el núcleo celular se ha convertido en uno de los focos principales de investigación. En particular, cómo las señales de $\mathrm{Ca}^{2+}$ que se generan en el citosol pueden afectar fenómenos nucleares. Asimismo, de un tiempo a esta parte, se ha comenzado a concebir al núcleo como un organelo capaz de generar y mantener su propia homeostasis de $\mathrm{Ca}^{2+}$ con relativa independencia de las variaciones en el $\mathrm{Ca}^{2+}$ citosólico (Hardingham et al., 1997; Itano et al., 2003; Echeverría et al., 2003). En esta línea de investigación, estamos estudiando la señalización a nivel nuclear por $\mathrm{Ca}^{2+}$ y el proceso de transcripción (figuras 2 y 3). Estamos utilizando como paradigma experimen- 
FIGURE 2. Carga de Ca2+ dependiente de ATP y sensibilidad a thapsigargin e ionomicina. Nucleaos aislados fueron incubados en un medio de reacción conteniendo: $1 \mathrm{mM}$ ATP, 20 iM GTP, CTP, UTP, 80 $\mathrm{mM} \mathrm{KCl}, 3 \mathrm{mM} \mathrm{NaCl}, 5 \mathrm{mM} \mathrm{MgCl} 2,50 \mathrm{mM}$ Tris-HCl, pH 7.4, $5 \mathrm{mM}$ oxalato de potasio y 1 ì Ca2+ libre suplementado con $45 \mathrm{Ca} 2+(0.5 \mathrm{iCi} / \mathrm{ml})$. La concentración de $\mathrm{Ca} 2+$ libre fue calculada de las constantes de asociación aparentes Ca-EGTA de acuerdo con Schwartzenbach, usando un programa de computación como descrito por Fabiato \& Fabiato, 1979. (A) Carga de calcio condición control (1 ì Ca2+ libre y $1 \mathrm{mM}$ ATP) (?), control más 10 ìM thapsigargin, TG ( ), o control más 2 ìM lonomycin (1, o 1 ìM Ca2+ libre sin ATP (?). (B) Movilización del 45Ca2+ por EGTA. Se dejaron a los nucleaos aislados cargar 45Ca2+ por 15 min y luego se adiciono $0.5 \mathrm{mM}$ EGTA al medio de reacción.

Imágenes microscopía confocal (C-E). Núcleos aislados fueron cargados con fluo-3, AM y tratados como descrito en (Benech et al, 2005). (C) 1 ìM Ca2+libre y $1 \mathrm{mM}$ ATP tiempo cero; (D) ìM Ca2+ libre y $1 \mathrm{mM}$ ATP a los 15 minutos; (E) $1 \mathrm{ìM}$ Ca2+libre y $1 \mathrm{mM}$ ATP a los 15 minutos, pero con 5ìM thapsigargin. Barra de escala: 5ìm. Reproducido con permiso de Elsevier Ltd.

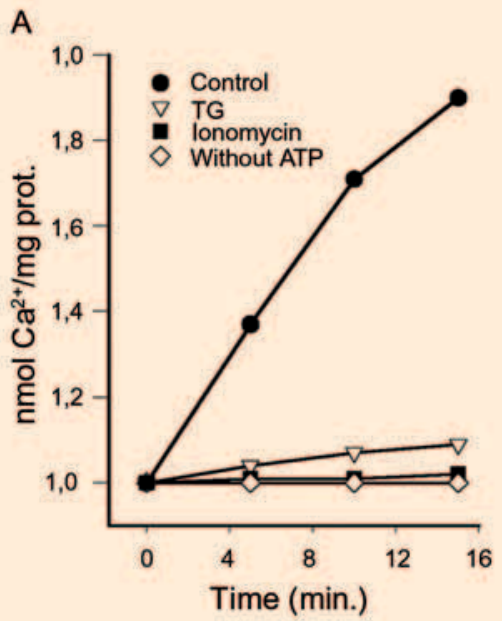

B
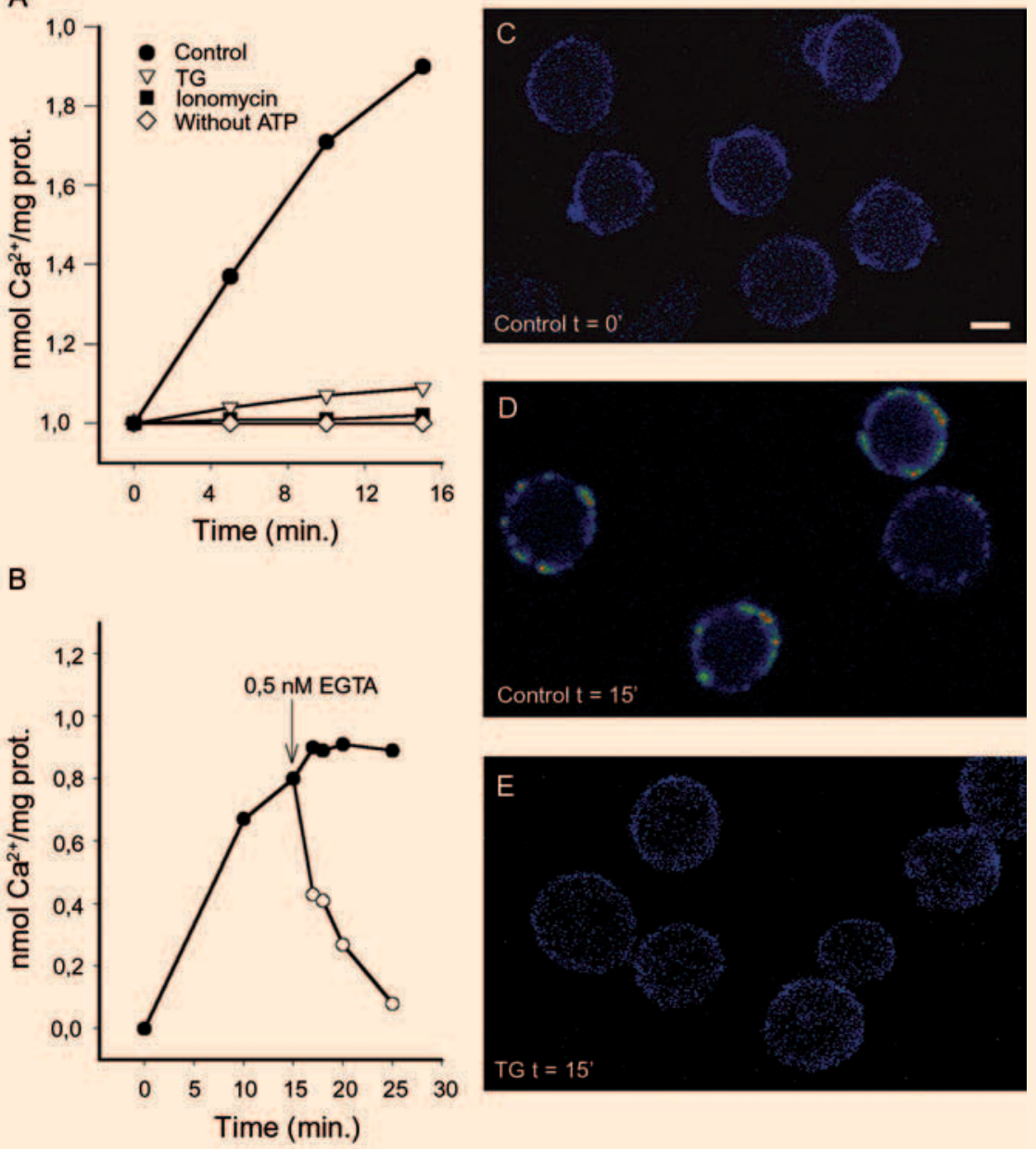

Time (min.) 
FIGURE 3. Modelo actualizado de señales de $\mathrm{Ca}^{2+}$ en el núcleo celular.

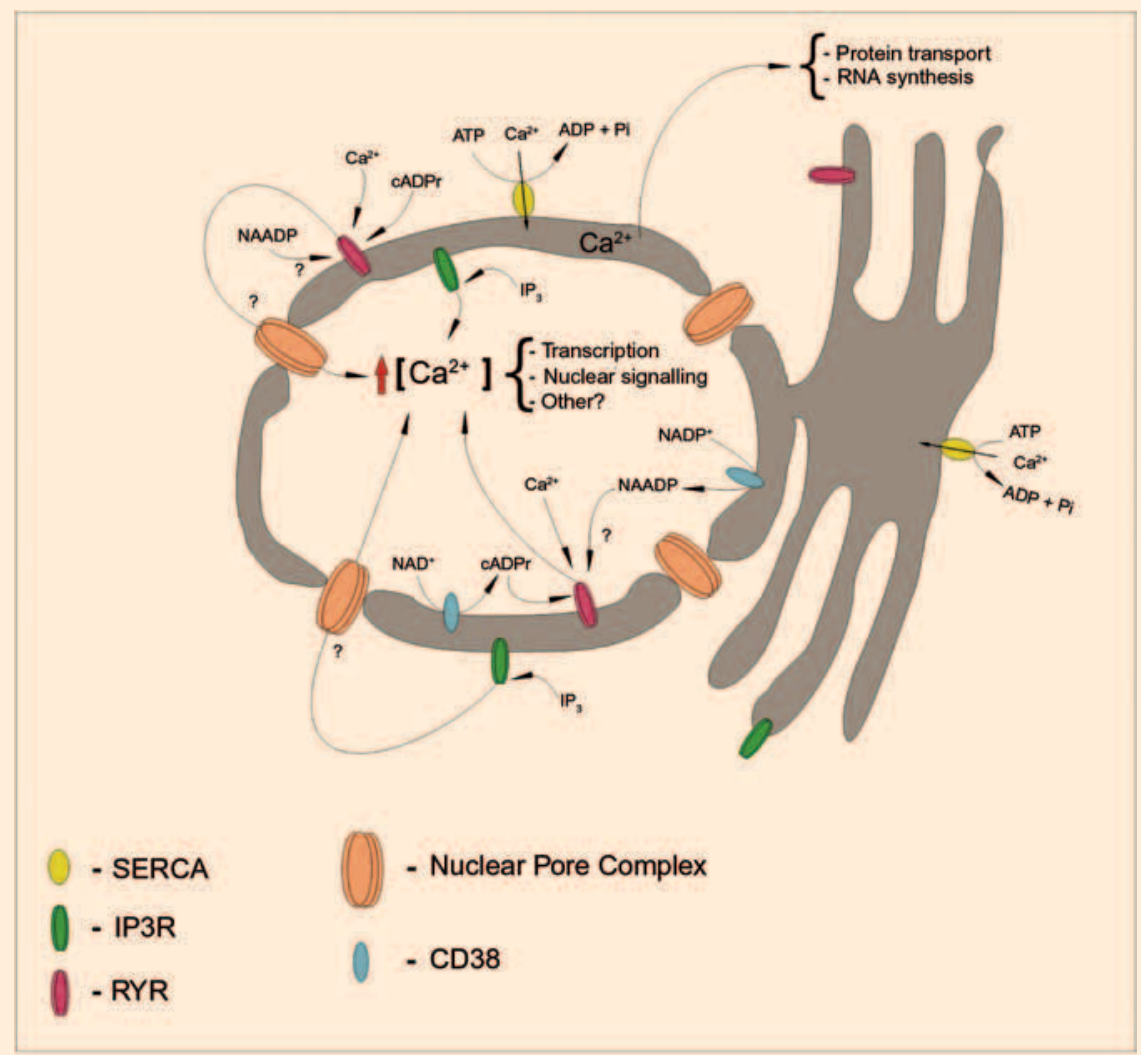

tal núcleos aislados de hígado de rata y células en cultivo (cultivo primario de células miometriales humanas y de neuronas). Hemos encontrado la existencia de por lo menos 2 componentes de $\mathrm{Ca}^{2+}$ que afectan la síntesis de ARN: a) concentración de $\mathrm{Ca}^{2+}$ en el nucleoplasma en sí misma; b) calcio acumulado en el envoltorio nuclear en forma dependiente de la bomba de calcio SERCA presente en dicho envoltorio (Benech et al. 2002, 2003 y 2005; Escande et al., 2007 a, b y c]. Hemos encontrado también, que la liberación de $\mathrm{Ca}^{2+}$ desde el envoltorio nuclear en respuesta a $\mathrm{IP}_{3}$ fue capaz de promover la fosforilación del factor de transcripción CREB y que un aumento del $\mathrm{Ca}^{2+}$ nuclear a $500 \mathrm{nM}$, promovió la activación de la transcripción del ARNm de PGC1-á (Escande, tesis de maestría, 2007). Esta proteína es un co-activador de la trascripción y en conjunto con CREB estimula la expresión de los mensajeros de PEPCK1 y G-6-Pasa, contribuyendo de esta forma a la regulación de la gluconeogénesis en hígado.

En conjunto, estos resultados sugieren que el núcleo celular es capaz de generar y mantener su propia homeostasis de $\mathrm{Ca}^{2+}$. Variaciones en la homeostasis de $\mathrm{Ca}^{2+}$ nuclear originadas en el propio núcleo celular, podría estar modulando procesos tan importantes como la transcripción. 


\section{ApLiCACIONES DE LOS DENDRÍMEROS A LA NANOBIOLOGÍA Y NANOMEDICINA}

\section{Dendrímeros y músculo liso}

Los dendrímeros son una clase única de vehículos nanoestructurados para la entrega de drogas y material genético a blancos intracelulares (Kolhe et al., 2003; Perumal et al., 2008). Como agentes de entrega de drogas, los dendrímeros presentan ventajas como la monodispersión y la multivalencia (Perumal et al., 2008; Cho et al., 2008). Se caracterizan por poseer un núcleo central y un gran número de grupos superficiales. Esto hace que moléculas pequeñas como drogas, enzimas o agentes de imagenología, puedan ser encapsulados o conjugados a los dendrímeros (Tassano et al., 2011; Hamoudeh et al., 2008). Estos polímeros han mostrado ser capaces de producir altos niveles intracelulares de droga y por lo tanto tienen un gran potencial terapéutico [He et al., 2011; Perumal et al., 2008). Asimismo, han demostrado un gran potencial como agentes de diagnóstico (Perumal et al., 2008). Su interacción con las membranas celulares y su internalización celular parecen depender del tamaño, generación y grupos funcionales de superficie (Perumal et al., 2008). En los últimos años, se han realizado varios estudios para evaluar los dendrímeros como transportadores de droga por vía oral [Sadekar y Ghandehari, 2012]. Por otra parte, ha sido observado que los dendrímeros PAMAM de la generación 4 redujeron los niveles de glucosa en sangre en ratas diabéticas [Karolczak , K. et al., 2012] y que dendrímeros modificados PAMAM-OH inhibieron la actividad de la $\mathrm{Na}^{+} / \mathrm{K}^{+}$ATPasa y $\mathrm{Ca}^{2+}$ ATPasa de eritrocitos humanos (Ciolkowski et al., 2011).

La interacción de los dendrímeros con las membranas celulares y la internalización celular parecen depender del tamaño, generación y grupos funcionales de superficie (Perumal et al. 2008). Particularmente, los grupos funcionales superficiales pueden producir una densidad de cargas altamente localizada, que puede tener una influencia importante en la interacción del dendrímero con la membrana celular y su internalización celular.

Nuestro grupo de investigación está estudiando las posibles aplicaciones de los dendrímeros en enfermedades que involucran al músculo liso (como el parto prematuro) y el cáncer.

El parto prematuro es la causa predominante de morbilidad y mortalidad en recién nacidos. Los nanomateriales actualmente en estudio pueden resultar útiles en cuanto al desarrollo de nuevas terapias para tratar los problemas fisiopatológicos de la contracción del músculo liso miometrial (que conllevan al parto prematuro). Para el desarrollo de estas terapias, el conocimiento de los mecanismos de captación celular de los distintos nanomateriales empleados por diferentes tipos celulares, se vuelve primordial. En este sentido, nuestro grupo está estudiando los mecanismos de captación de un nanoconjugado en cultivos primarios de células miometriales humanas (CMH). El nanoconjugado, G4-FITC, fue obtenido al marcar dendrímeros de poliamidoamina (PAMAM) de la generación 4 (G4) con fluoresceína isotiocianato (FITC).

El nanoconjugado obtenido fue caracterizado usando HPLC, NMR y microscopía de fuerza atómica. La concentración de nanoconjugado empleada en los experimentos $(7 \mu \mathrm{g} / \mathrm{ml})$, mostró no afectar significativamente la viabilidad de las $\mathrm{CMH}$ hasta las 48 h. El análisis por microscopia láser confocal de CMH incubadas con G4-FITC mostró señal de fluorescencia a nivel perinuclear a partir de las 5 h o más (24, 36 y 48 h) de incubación. A las 24 h, G4-FITC co-localizó parcialmente con "Lysotracker" (figura 4), un compuesto fluorescente capaz de marcar organelos acídicos en células vivas 
FIGURA 4. Co-localización parcial de G4-FITC y Lysotracker. Las HMC fueron incubadas por 24 h con G4-FITC y luego $1 \mathrm{~h}$ con Lysotracker. Las imágenes se obtuvieron por LCM. G4-FITC (A), Lysotracker (B), Merge (C). Magnificación: $180 \mathrm{x}$, las barras de escala indicando un tamaño de $10 \mu \mathrm{m}$. La longitud de onda de excitación fue de $488 \mathrm{~nm}$ y $543 \mathrm{~nm}$ para FITC y Lysotracker, respectivamente. Las imágenes fueron superpuestas mediante el software del sistema LCM para observar co-localización.
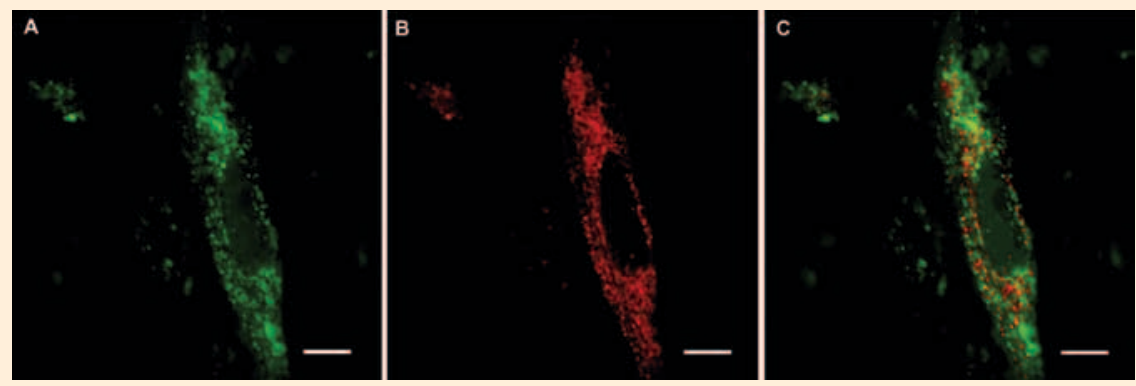

FIGURA 5. El G4-FITC ingresa en núcleos aislados de HMC e hígado de rata. Los núcleos aislados fueron incubados por $1 \mathrm{~h}$ con G4-FITC y observados por LCM. Núcleos aislados de HMC: G4-FITC (A), DAPI (B), Merge (C). Núcleos aislados de hígado de rata: G4-FITC (D). Magnificación: $120 \mathrm{x}$. Las barras de escala indican un tamaño de $10 \mu \mathrm{m}$ (imágenes a, b, y c). Magnificación: 100 x. Las barras de escala indican un tamaño de $20 \mu \mathrm{m}$ (imagen d).

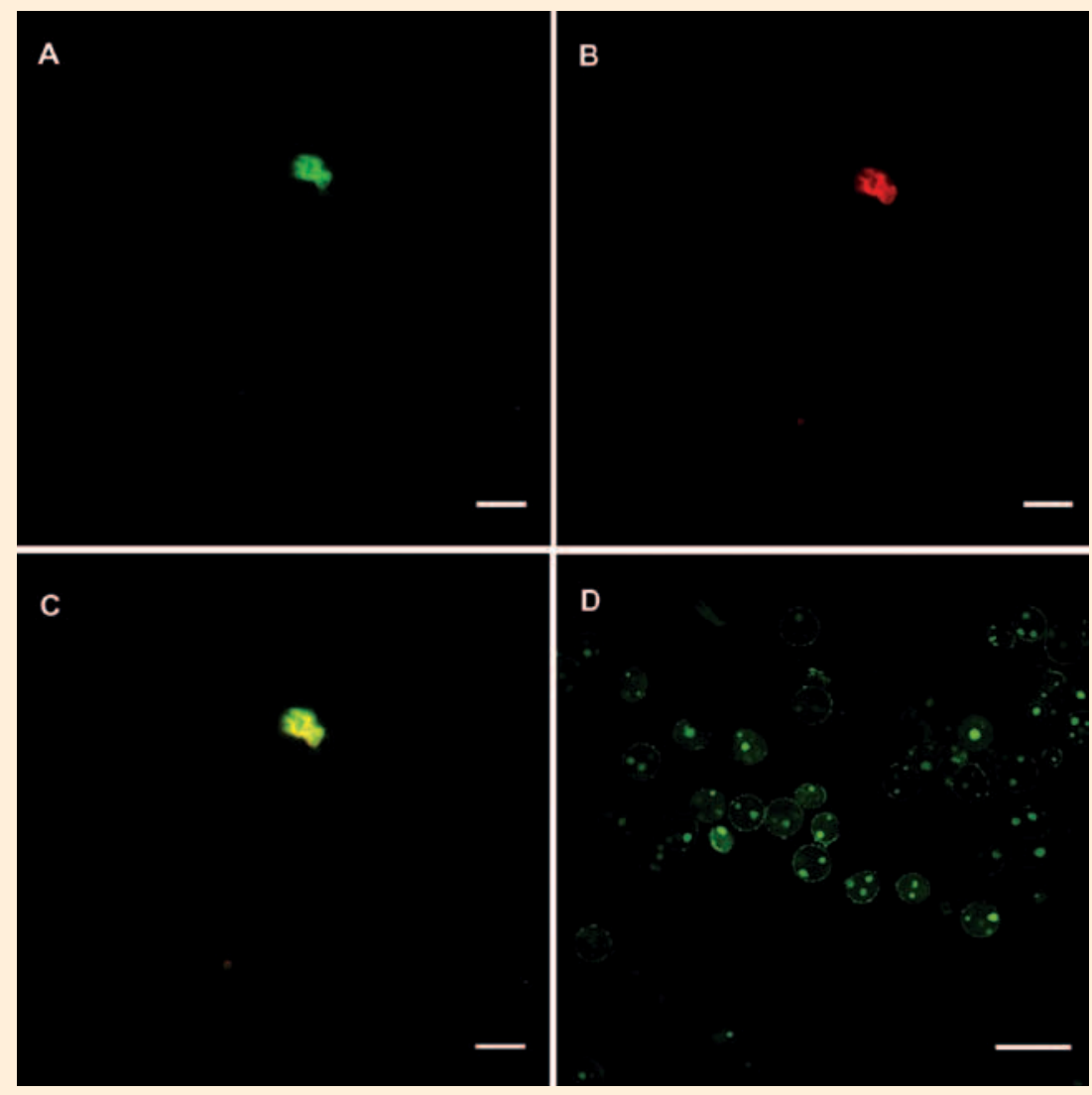


(lisosomas). Por otra parte, utilizando diferentes tipos de inhibidores de endocitosis y citometría de flujo, logramos identificar el mecanismo de ingreso del nanoconjugado a las CMH. De acuerdo con nuestros resultados, en CMH en cultivo, el G4-FITC estaría siendo captado por medio de endocitosis dependiente de clatrina. Esta conclusión, se basa en el hecho de que la clorpromazina (inhibidor de la endocitosis mediada por clatrina), inhibió la captación de transferrina (control positivo de endocitosis mediada por clatrina) y G4-FITC de forma significativa. La filipina (inhibidor de la endocitosis mediada por caveolina), sin embargo, no mostró tener un efecto inhibitorio significativo en la captación de BODIPY-LacCer (control positivo de endocitosis mediada por caveolina) ni de G4-FITC. Es interesante destacar, que en $\mathrm{CMH}$, nunca detectamos señal de fluorescencia del nanoconjugado caracterizado (G4-FITC) a nivel del núcleo celular. Sin embargo, cuando electroporamos CMH en presencia de G4-FITC, detectamos fluorescencia a nivel de algunos núcleos de CMH. Comprobamos experimentalmente que núcleos aislados de CMH incubados con G4-FITC presentaron señal de fluorescencia (figura 5). Esta serie de resultados sugieren que los G4-FITC en $\mathrm{CMH}$ estarían siendo internalizados por endocitosis dependiente de clatrina, con localización lisosomal a las 24 h. Por otra parte, los resultados también sugieren que la electroporación modifica la distribución intracelular del G4-FITC en este tipo celular, pudiendo convertirse en una tecnología interesante para electrotransferir drogas al citosol o al núcleo de células de músculo liso (Oddone, N. et al., 2013).

\section{Dendrímeros y cáncer}

El efecto EPR (efecto del incremento de la permeabilidad y retención) se basa en las características fisiopatológicas únicas de los tumores sólidos, las cuales otorgan una ventaja al uso de nanopartículas como vehículos de agentes terapéuticos. Este efecto, surge como consecuencia de la angiogénesis extensa que resulta en la hiper-vascularización, drenaje linfático limitado y aumento de la permeabilidad a los lípidos y macromoléculas. Estas características, ayudan a asegurar el suministro adecuado de nutrientes para satisfacer las necesidades metabólicas de los tumores de crecimiento rápido (Heuser et al., 1986; Maeda et al., 2000).

El abordaje experimental que emplea nanopartículas tradicionales polidispersas, como por ejemplo liposomas y polímeros convencionales, mostró serias complicaciones. Afortunadamente este problema (la capacidad de ajustar tamaños exactos y uniformes) puede ser abordado con éxito empleando dendrímeros, ya que es posible la selección de una entidad de tamaño exacto (Choi y Baker, 2005). La capacidad de construir poblaciones monodispersas de dendrímeros en el rango de tamaño necesario para aprovechar el efecto EPR, es un paso alentador hacia la explotación de las propiedades tumorales como estrategia de captación pasiva de los dendrímeros. En un trabajo reciente de nuestro grupo (Tassano et al., 2011), se logró marcar los dendrímeros PAMAM G4-FITC con el intermediario [ $\left.{ }^{99 \mathrm{~m}} \mathrm{Tc}(\mathrm{CO}) 3(\mathrm{H} 2 \mathrm{O}) 3\right]+$ con una pureza radioquímica de $\sim 90 \%$. Desarrollamos un método de análisis por HPLC usando columnas C18 y encontramos que el compuesto fue estable por 24 horas. En ratones con melanoma inducido, observamos que el patrón de biodistribución del conjugado fue similar al encontrado en ratones normales. Sin embargo, a la hora post-inyección, observamos una alta captación del conjugado por parte del tumor. La captación tumoral, fue 4-10 veces mayor que la captación observada por la musculatura circundante, lo que permitió un buen contraste y la obtención de buenas imágenes cente- 
llográficas (figura 6). La elevada captación por parte del tumor puede ser explicada por el efecto EPR descrito anteriormente. El análisis de las imágenes por microscopía confocal, mostraron que el ${ }^{99 \mathrm{~m}} \mathrm{Tc}(\mathrm{CO}) 3$-dendrímero-FITC fue internalizado por las células del hígado y del tumor, con señal fluorescente detectada a nivel del citoplasma celular (figura 7). Los estudios biológicos mostraron el potencial del compuesto desarrollado en la obtención de imágenes moleculares oncológicas (Tassano et al., 2011). Por otra parte, nuestro grupo estudió la biodistribución de ${ }^{99 \mathrm{~m} T c}$ (CO)3-dendrímero en otro modelo de tumor murino. Dicho estudio fue realizado en ratones Balb-

FIGURA 6. Imagen centellográfica de ratón normal (A) y ratón portador de melanoma (B) inyectados con ${ }^{99 \mathrm{~m} T C}$ (CO)3-dendrímero-FITC, $1 \mathrm{~h}$ post-inyección. (A) Las flechas blancas y amarillas muestran el hígado y los riñones, respectivamente. (B) En blanco se muestra la región donde se localiza el tumor. En amarillo se muestra la región abdominal (hígado y riñones) sobre la cual se colocó una máscara de de manera que no interfiera con la imagen de la región del tumor.
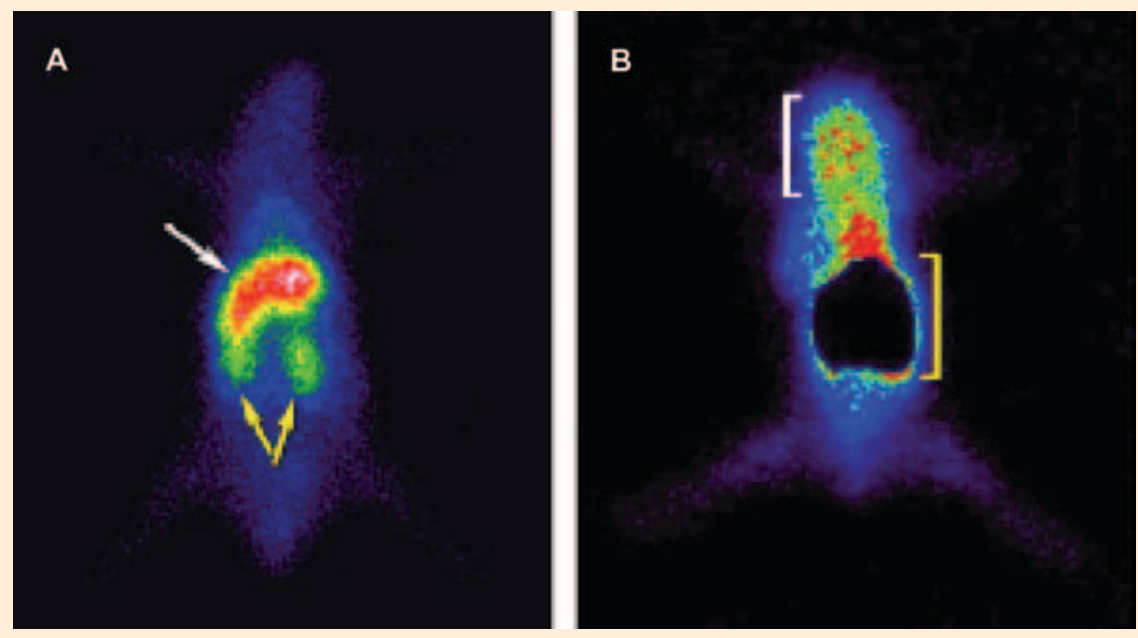

FIGURA 7. Imágenes de microscopía láser confocal: secciones de hígado (A) y tumor (B) provenientes de ratones portadores de melanoma inyectados con ${ }^{99 \mathrm{~m} T C}$ (CO)3-dendrímero-FITC, 24 h post- inyección
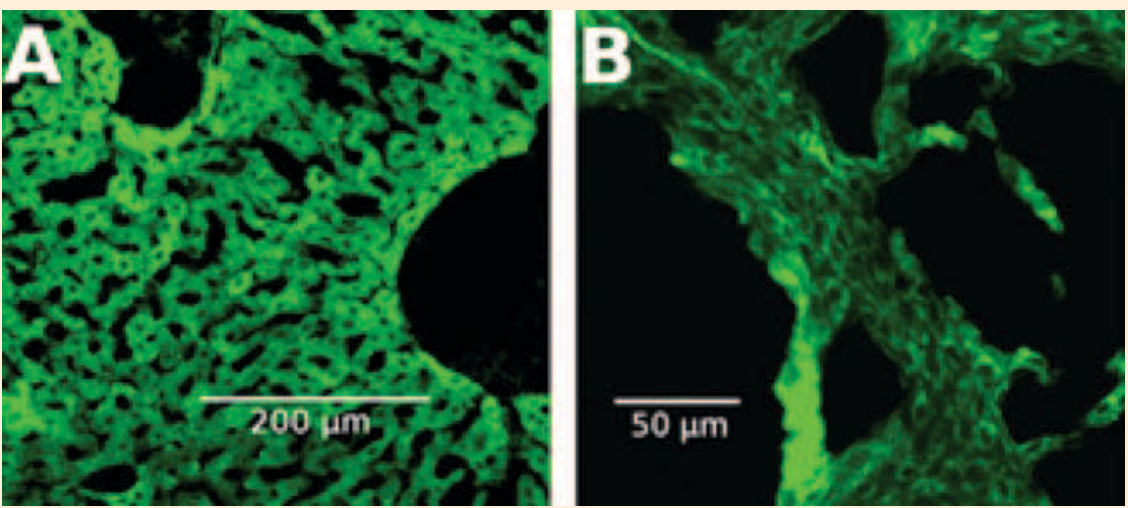
c con tumor mamario inducido con células $4 \mathrm{~T} 1$. La biodistribución empleando ${ }^{99 \mathrm{~m}} \mathrm{Tc}$ (CO) 3-dendrimero en ratón con tumor mamario fue similar a la observada en ratones normales, con una captación significativa, 1 h post-inyección. Los resultados están expresados en \% actividad/g a los tiempos 1 h y 3 h, respectivamente. Se observó acumulación hepática $(21.9 \pm 3.4,16.4 \pm 3.3)$, renal $(27.9 \pm 10.2,12.8 \pm 0.9)$ y tumoral $(1.39 \pm 0.2,0.77 \pm 0.1)$. A nivel del tumor, el \% actividad/g fue de 3-4 veces mayor que en el músculo circundante donde el \% actividad/g fue: $0.4 \pm 0.05$ y $0.3 \pm 0.2$ a los tiempos 1 h y $3 \mathrm{~h}$, respectivamente.

En este modelo, también se inyectó dendrímero-FITC en forma intravenosa. Se evaluó la captación por las células del tumor a las 24 h post-inyección por microscopía láser confocal. Se observó señal de fluorescencia en el citosol de las células tumorales.

\section{MicrosCopía de FUERZA ATÓMICA EN CIENCIAS BIOLÓGICAS Y BIOMÉdICAS}

La microscopía de fuerza atómica (MFA) va emergiendo como una herramienta muy poderosa en la investigación en general y particularmente aplicada a las ciencias biológicas y biomédicas. La MFA permite el análisis topográfico y mecánico de todo tipo de materiales (conductores y no conductores) a escala nanométrica. El MFA de nuestro instituto tiene acoplado un microscopio óptico invertido de epifluorescencia, el cual posibilita la combinación de imágenes topográficas con el marcaje de células y tejidos utilizando colorantes, sondas o anticuerpos específicos. Permite el análisis topográfico y mecánico a escala nanométrica de material biológico vivo.

Las posibilidades para la investigación en el área biológica y biomédica son múltiples permitiendo:

1. Imagenología de células vivas:
a) Identificación de biomoléculas y estructuras celulares.
b) Observación en tiempo real de eventos de señalización celular.
c) Estudios de farmacología in situ.
d) Observación de eventos de interacciones celulares y adhesión celular.

2. Imagenología de alta resolución de moléculas:
a) ADN y plásmidos.
b) Estructura de biopolímeros.
c) Imagenología de membranas biológicas.
d) Combinación de experimentos de fluorescencia con MFA.

3. Estudios funcionales:
a) Medidas de elasticidad de membranas celulares.
b) Espectroscopía de fuerza, volumen.
c) Nano-manipulación.
d) Estudio de plegamiento de proteínas.
e) Estudio de interacciones receptor-ligando.

Por revisión reciente ver Whited, A. M \& Park, P. S.-H., 2013.

Utilizando MFA, estamos obteniendo imágenes de alta resolución estudiando el proceso de internalización celular de nanocompuestos como dendrimeros y SPIONs 
FIGURA 8. Imágenes de microscopía de fuerza atómica de CMH incubada con G4-FITC y fijada con PFA 3\%. (A) Imagen de altura $\mathrm{CMH}$. (B) Imagen de deflexión $\mathrm{CMH}$. (C) Imagen de deflexión de región de CMH donde se observan acúmulos de G4-FITC sobre la misma. Las barras de escala indican un tamaño de $15 \mu \mathrm{m}$ (A y B). La barra de escala indica un tamaño de $1 \mu \mathrm{m}$ (C).
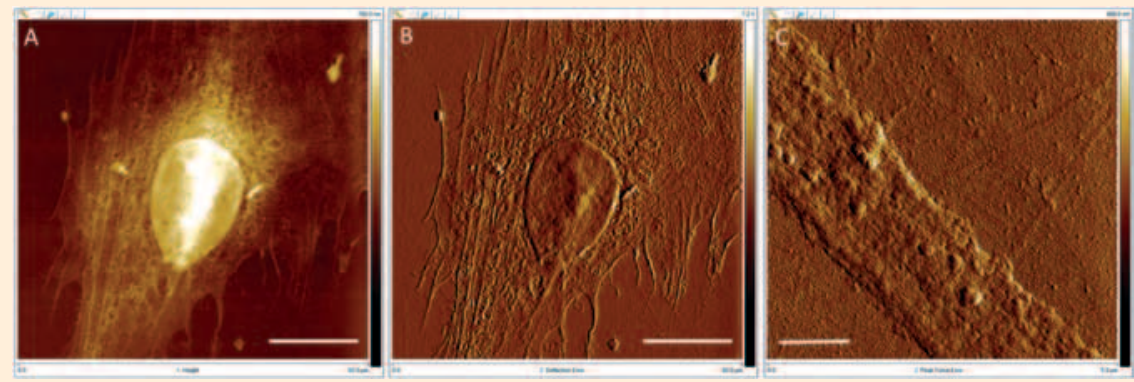

(Superparamagnetic iron oxide nanoparticles) en diferentes cultivos de líneas celulares o cultivos primarios como las $\mathrm{CMH}$ mencionadas anteriormente (figura 8).

También estamos estudiando por AFM las propiedades materiales de cardiomiocitos vivos obtenidos de ratones control o diabéticos. La prevalencia de la diabetes mellitus (DM) está creciendo rápidamente. Ha sido estimado que el número de personas adultas afectadas por la diabetes a nivel global aumentará de 171 millones en el 2000 a 300 millones en el 2030 (Wild et al., 2000). La DM es un factor de riesgo bien reconocido por desarrollar insuficiencia cardíaca. De hecho, el Framingham heart stu$d y$ ha mostrado que la frecuencia de insuficiencia cardíaca es 2 veces mayor en hombres diabéticos y 5 veces mayor en mujeres diabéticas en comparación con sujetos controles de la misma edad (Kannel y McGee, 1979). Por lo tanto, complicaciones cardiovasculares son la causa principal de morbilidad y mortalidad relacionada con la diabetes (Garcia et al., 1974).

La DM es responsable de diversas complicaciones cardiovasculares como aumento en la arteriosclerosis en las grandes arterias y el corazón, lo que aumenta el riesgo

FIGURA 9. Imágenes de microscopía de fuerza atómica de cardiomiocito aislado fijado en PFA 3\%. (A) Imagen de altura. (B) Imagen de deflexión. Las barras de escala indican un tamaño de 5 um (A y B).
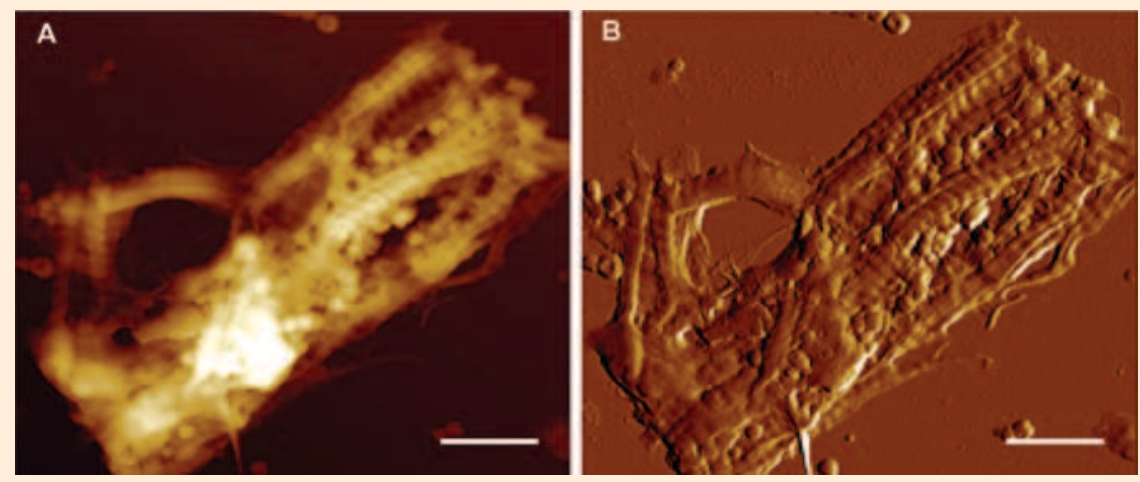
de infarto de miocardio (Boudina y Abel 2007). En modelos animales de diabetes, varias alteraciones funcionales y estructurales del corazón han sido documentadas tanto en diabetes tipo I como tipo II. En diabetes tipo I, la mayoría de los estudios se han realizado en animales en los cuales la diabetes se induce por administración de estreptozotocina (droga que elimina las células beta del páncreas) (Joffe II et al., 1999; Nielsen et al., 2002; Boudina y Abel, 2007). Nuestro grupo de investigación está trabajando con ratones CD1, a los cuales se les induce diabetes tipo I por inyección intraperitoneal de la droga antes mencionada. Hemos constatado un aumento en la muerte de ratones diabéticos en comparación con ratones control de la misma edad. Como mencionado anteriormente, el MFA permite medir y conocer las propiedades materiales dinámicas visco-elásticas de las células vivas. Las medidas de elastografía que se realizan por MFA, permiten mapear la distribución espacial de las propiedades mecánicas de la célula. Estas propiedades mecánicas son reflejo de la estructura celular. A través de las medidas obtenidas, sería posible detectar cambios promovidos por varias enfermedades a nivel de célula única. Estos cambios han sido observados en varios tipos celulares y enfermedades diferentes (Somer y Meiselman, 1993; Hansma y Hoh, 1994; Wu et al., 2000; Alexopoulos et al., 2003; Shelby et al., 2003; Costa, 2004; Perrault et al., 2004). Los resultados preliminares obtenidos con cardiomiocitos vivos aislados de corazones de ratones diabéticos o de ratones control mostraron que el módulo elástico aparente medido por MFA resultó un 100\% mayor en los cardiomiocitos diabéticos. Estos resultados sugieren que los cardiomiocitos de los ratones diabéticos son más duros que los provenientes de ratones control. Los resultados sugieren que la diabetes, estaría modificando las propiedades mecánicas de los cardiomiocitos, aspecto que abordaremos próximamente.

\section{CONCLUSIÓN}

La base de datos actual de clasificación de toxicidad de una sustancia se basa en el hecho de que una vez probada la toxicidad de la misma, todas las sustancias similares serán clasificadas como tóxicas. Este mismo razonamiento aplica para sustancias no tóxicas. Sin embargo, esta lógica no puede ser aplicada en el caso de sustancias formuladas a nanoescala, pues aquí intervienen otro tipo de elementos tales como tamaño, área de superficie, morfología de las partículas, composición química, reactividad, entre otros aspectos.

Hoy contamos con numerosos sistemas nanoestructurados nuevos con múltiples aplicaciones potenciales en el área biomédica. Es necesario, además de la caracterización físicoquímica de estos materiales (tamaño de partículas, potencial zeta, solubilidad, estabilidad, etc.), estudiar su interacción con los sistemas biológicos. Asimismo, conocer la capacidad de estos sistemas nanoestructurados de ingresar en las células, su mecanismo de ingreso, su posible metabolización y su interacción con nanobiomoléculas. Y, desde luego, conocer también si puede provocar daño a nivel celular o del organismo y caracterizar el tipo de daño. Un conocimiento detallado de la interacción de los sistemas nanoestructurados con los sistemas biológicos nos conducirá al desarrollo de sistemas nanoestructurados biocompatibles. Este conocimiento permitirá desarrollar nuevas terapias para el tratamiento de enfermedades como parto prematuro, cáncer, enfermedades neurodegenarativas, diabetes, por mencionar algunas. 


\section{REFERENCIAS BIBLIOGRÁFICAS}

Alexopoulos, L. G.; M. A. Haider, T. P. Vail y F. Guilak (2003) "Alterations in the mechanical properties of the human chondrocyte pericellular matrix with osteoarthritis". J. Biomech. Eng. 125: 323-333.

Alvarez, J., Giuditta, A. y Koenig, E. (2000) "Protein synthesis in axons and terminals: Significance for maintenance, plasticity and regulation of phenotype. With a critique of slow transport theory". Progress in Neurobiol. 62: 1-62.

Balogh, L.P. 2010. Why do we have so many definitions for nanoscience and nanotechnology? Nanomedicine. 6: 397-398.

Benech J.C.; Galina A. y de Meis, L. (1991) “Correlation between $\mathrm{Ca}^{2+}$ uptake, $\mathrm{Ca}^{2+}$ efflux and phosphoenzyme level in sarcoplasmic reticulum vesicles”. Biochem J. 274: 427-432.

Benech J.C.; Wolosker H. y de Meis, L. (1995) "Reversal of the calcium pump of blood platelets". Bichem. J. 306: 35-38.

Benech J.C.; Crispino, M.; Chun, J.T.; Kaplan, B.B. y Giuditta, A. (1994) “Protein synthesis in nerve endings from squid brain: Modulation by calcium ions”. Biol. Bull. 187: 269.

Benech J.C.; Crispino, M.; Martin, R.; Álvarez, J.; Kaplan, B.B. y Giuditta, A. (1996) "Protein synthesis in the presynaptic endings of the squid photoreceptor neuron: In vitro and in vivo modulation". Biol. Bull. 191: 263.

Benech, J.C.; Crispino, M.; Kaplan, B.B. y Giuditta, A. (1997) "Protein synthesis in presynaptic endings of squid brain: Regulation by $\mathrm{Ca}^{2+}$ ions", en: J.R. Sotelo y J. C. Benech (eds.). Calcium and cellular metabolism: Transport and regulation. Plenum Press, Nueva York, Londres: 155-162.

Benech, J.C.; Crispino, M.; Kaplan, B.B. y Giuditta, A. (1999) "Protein synthesis in presynaptic endings from squid brain: Modulation by calcium ions". Journal. Neurosc. Res. 55: 776-781.

Benech, J.C:, Lima, P., Sotelo, J.R. y Brown, E. (2000) “ $\mathrm{Ca}^{2+}$ dynamics in synaptosomes isolated from the squid optic lobe". Journal. Neurosc. Res. 62: 840-846.

Benech, J.C., Escande, C. y Sotelo, J.R. (2002) "Effect of the SERCA Ca ${ }^{2+A T P a s e}$ inhibitor thapsigargin and $\mathrm{Ca}^{2+}$ ionophores on RNA synthesis and over ATP dependent $\mathrm{Ca}^{2+}$ uptake in isolated rat liver nuclei". XIV International Biophysics Congress, Argentina 2002.

Benech, J.C.; Escande, C. y Sotelo, J.R. (2003) “Correlation between RNA synthesis and the $\mathrm{Ca}^{2+}$ filled state of the nuclear envelope store. International Workshop 'Calcium release and cellular calcium signalling domains'” septiembre 28 octubre 2, 2003. Marbella, Chile.

Benech, J.C.; Escande, C. y Sotelo, J.R. (2005) “Correlation between RNA synthesis and the $\mathrm{Ca}^{2+}$ filled state of the nuclear envelope store". Cell Calcium, 38 (2): 101-109.

Calliari, A.; Sotelo-Silveira, J.; Costa, M.C.; Nogueira, J.; Cameron, L.C.; Kun, A.; Benech, J.C.; Sotelo, J.R. (2002) Cell Mot. and Cytosk. 51, 169-76

Boudina, S y Abel, L.D. (2007) Diabetic cardiomyopathy revisited. 2007. Circulation 115: 3213-3223

Cho, K.; Wang, X.; Nie, S.; Chen, Z.G.; Shin, D.M. (2008) “Therapeutic nanoparticles for drug delivery in cancer". Clin Cancer Res, 14 (5): 1310-1316

Choi, Y. y Baker Jr., J. R. (2005) “Targeting cancer cells with DNA-assembled dendrimers: mix and-match strategy for cancer". Cell Cycle, 4: 669-671. 
Costa, KD. (2004) "Single-cell elastography: Probing for disease with the atomic force microscope". Desease markers, 19: 139-154.

Ciolkowski, M.; Rozanek, M.; Szewczyk, M.; Klajnert, B. y Bryszewska, M. (2011) “The influence of PAMAM-OH dendrimers on the activity of human erythrocytes ATPases". Biochim. Biophys. Acta. 1808: 2714-2723.

Crispino, M.; Castigli, E.; Perrone Capano, C; Martin, R.; Menichini, E.; Kaplan, B.B. y Giuditta, A. (1993) "Protein synthesis in a synaptosomal fraction from squid brain" Mol. Cell. Neurosci, 4: 366-374.

Crispino, M.; Kaplan, B.B.; Martin, R.; Alvarez, J.; Chun, J.T.; Benech, J.C. y Giuditta A. (1997) "Active polysomes are present in the large presynaptic endings of the synaptosomal fraction from squid brain". J. of Neurosc, 17: 7694-7702.

Echevarria, W.; Leite, M.; Guerra, M.; Zipfel, W.; Nathanson, M. (2003) Nature Cell Biology, 5: 440-446.

Escande, C.; Arbildi, P.; Chini, E. y Benech, J.C. (2007a) “The nuclear envelope store and the regulation of transcription". En: Alex R. Demesi (ed.) Cellular signaling and apoptosis research. Nova Science Publishers. NY. ISBN. 1-60021-565-3: 201-219.

Escande, C., Arbildi, P.; Chini, E. y Benech, J.C. (2007b) The nuclear envelope store and the regulation of transcription". En: Nickolas 0. Grachevsky (ed.) Signal transduction research trends. ISBN. 1-60021-847-8: 187-205.

Escande, C.; Arbildi, P.; Chini, E. y Benech, J.C. (2007c) “A rise in nucleoplasmic Ca2+ can modulate CREB phosphorylation and the expression of the mRNA of the transcriptional co-activator PGC1-á in isolated liver nuclei". P.022, ICBP. Montevideo, Uruguay.

Escande, C. (2007) Regulación de la expresión génica por señales de calcio en el hígado. Efecto de la variación de Ca2+ nuclear en la fosforilación del factor de transcripción CREB y en la expresión del ARN mensajero de PGC1-alfa. Tesis de maestría (PEDECIBA). Abril, 2007.

Fabiato, A. y Fabiato, F. (1979) “Calculator programs for computing the composition of the solutions containing multiple metals and ligands used for experiments in skinned muscle cells". J. Physiol. (Paeis), 75: 463-505.

Garcia, M.J.; McNamara, P.M.M Gordon, T. et al. (1974) "Morbidity and mortality in diabetics in the Framingham population: Sixteen year follow-up study". Diabetes, 23: 105-111.

García Teijeiro, R.; Sotelo Silveira, J.R.; Sotelo, J.R. y Benech, J.C. (1999) “Calcium efflux from platelet vesicles of the dense tubular system. Analysis of the possible contribution of the $\mathrm{Ca}^{2+}$ pump". Molec. and Cell. Biochem, 199: 7-14.

Hamoudeh, M.; Kamleh, M.A.; Diab R.; Fessi, H. (2008) "Radionuclides delivery systems for nuclear imaging and radiotherapy of cancer". Advanced drug delivery reviews, 60 (12): 1329-1346

Hardingham, G.; Arnold, F.; Bading, H. (2001) Nature Neuroscience, 4(3): 261-267.

He, H.; Li, Y.; Jia, X.R.; Du, J.; Ying, X.; Lu, WL.; Lou, J.N.; Wei, Y. (201) "PEGylated Poly(amidoamine) dendrimer-based dual-targeting carrier for treating brain tumors". Biomaterials, 32 (2): 478-487

Heuser, L.S. y Miller, F.N. (1986) “Differential macromolecular leakage from the vasculature of tumors". Cancer, 57: 461-464.

Hansma, H.G. y Hoh, J.H. (1994) "Biomolecular imaging with the atomic force microscope". Annual review of biophysics and biophysical chemistry, 23: 115-139. 
Itano, N.; Okamoto, S.; Zhang, D.; Lipton, S.A.; Ruoslahti, E. (2003) Proc. Natl. Acad. Sci. U. S. A. 29, 100 (9): 5181-5186.

Jain, R. K. (1994) “Barriers to drug-delivery in solid tumors”. Sci. Am, 271: 58-65.

Jain, K.K. (2012) The handbook of Nanomedicine. Humana Press: 1-538.

Joffe, II.; Travers, KE.; Perreault-Micale, C.L. et al. (1999) "Abnormal cardiac function in the streptozotocin-induced non-insulin-dependent diabetic rat: Noninvasive assessment with Doppler echocardiography and contribution of the nitric oxide pathway". J Am Coll Cardiol, 34: 2111-2119.

Kannel, W.B, McGee, D.L. (1979) "Diabetes and cardiovascular disease: The Framingham study". JAMA, 241: 2035-2038.

Karolczak, K.; Rozalska, S.; Wieczoreck, M.; Labieniec-Watala, M. y Watala, C. (2012) "Poly(amido)amine dendrimers generation 4.0 (PAMAM G4) reduce blood hyperglycaemia and restore impared blood-brain barrier permeability in streptozotocin diabetes in rats". Int. Jour. Pharmaceutics, 436: 508-518.

Koenig, E. y Giuditta, A. (1999) "Protein synthesis machinery in the axon compartment". Neurosci, 89: 5-15.

Kolhe, P.; Misra E.; Kannan, R.M.; Kannan, S.; Lieh-Lai, M. (2003) “Drug complexation, in vitro release and cellular entry of dendrimers and hyperbranched polymers". Int J Pharm 259 (1-2): 143-160. doi: S0378517303002254 [pii].

Kroll, A. (2012) "Nanobiology-convergence of disciplines inspires great applications". Cell Mol Life Sci. 69: 335-336.

Maeda; H. Wu, J. Sawa, T. Matsumura, Y. Hori. K. (2000) “Tumor vascular permeability and the EPR effect in macromolecular therapeutics: A review" J. Controlled Release, 65: 271-284.

Nielsen, L.B.; Bartels, E.D.; Bollano, E. (2002) “Overexpression of apolipoprotein B in the heart impedes cardiac triglyceride accumulation and development of cardiac dysfunction in diabetic mice". J Biol Chem, 277: 27014-27020.

Ochs, S. (1982) Axoplasmic transport and its relation to other nerve functions. Nueva York: Wiley.

Oddone, N.; Zambrana, A.I.; Tassano, M.; Porcal, W.; Cabral, P. y Benech, J.C. (2013) “Cell uptake mechanisms of PAMAM G4-FITC dendrimer in Human Myometrial”. Cells Journal of Nanoparticle Research. En prensa.

Perrault, C. M.; Bray, E. J. Didier, N. Ozaki, C. K. y Tran-Son-Tay. R. (2004) "Altered rheology of lymphocytes in the diabetic mouse". Diabetologia, 47: 1722-1726.

Perumal, O.P., Inapagolla, R.; Kannan, S.; Kannan, R.M. (2008) "The effect of surface functionality on cellular trafficking of dendrimers". Biomaterials, 29 (24-25): 3469-3476.

Sadekar, S. y Ghandehari, H. (2012) “Transepithelial transport and toxicity of PAMAM dendrimers: Implications for oral drug delivery”. Adv. Drug. Deliv. Rev., 64: 571-588.

Somer, T. y H. J. Meiselman. (1993) "Disorders of blood viscosity". Ann. Med, 25: 31-39.

Shelby, J. P.; White, J. Ganesan, K. Rathod, P. K. y Chiu. D. T. (2003) "A microfluidic model for single-cell capillary obstruction by Plasmodium falciparum-infected erythrocytes". Proc. Natl. Acad. Sci. USA; 100:14618-14622.

Sotelo, J.R. y Benech, J.C. (1997) “Calcium and cellular metabolism”. Plenum Press. Nueva York, Londres: 125-142. 
Sotelo, J.R.; Kun, A.; Benech, J.C.; Giuditta, A.; Morillas, J. y Benech, C.R. (1999) “Ribosomes and Polyriebosomes are present in the squid giant axon: An inmunocytochemical study". Neuroscience, 90: 705-715.

Sotelo-Silveira, J.R.; Calliari, A.; Kun, A.; Benech, J. C.; Sanguinetti, C.; Chalar, C. y Sotelo, J.R. (2000) "Neurofilament mRNAs are present and translated in the normal and severed sciatic nerve". Journal. Neurosc. Res, 62: 65-74.

Tassano, M.R.; Audicio, P.F.; Gambini, J.P.; Fernandez, M.; Damian, J.P.; Moreno, M.; Chabalgoity, J.A.; Alonso, O.; Benech, J.C.; Cabral, P. (2011) "Development of 99mTc(CO) (3)-dendrimer-FITC for cancer imaging". Bioorganic \& medicinal chemistry letters, 21 (18): 5598-5601.

Whited, A. M. y Park, P. S.-H. (2013) "Atomic force microscopy: A multifaceted tool to study membreane proteins and their interactions with ligands". Biochim. Biophys. Acta. $<$ http://dx.doi.org/10.1016/j.bbamem.2013.04.011>.

Wild, S.; Roglic, G.; Green, A. et al. (2004) “Global prevalence of diabetes: Estimates for the year 2000 and projections for 2030". Diabetes Care, 27(5): 1047-53.

Wu, Z. Z.; Zhang, G. Long, M. Wang, H. B. Song, G. B. y Cai. S. X. (2000) “Comparison of the viscoelastic properties of normal hepatocytes and hepatocellular carcinoma cells under cytoskeletal perturbation". Biorheology, 37: 279-290. 